\title{
Avaliação e correção automática no software livre RStudio
}

\author{
Thaísa Jacintho Müller - thaisa.muller@pucrs.br - PUCRS \\ Filipe Jaeger Zabala - filipe.zabala@pucrs.br - PUCRS
}

\begin{abstract}
Resumo: Este artigo fornece embasamento para a criação de documentos para avaliação e correção automática de questões, exemplificado no relato de uma experiência realizada em um curso de extensão sobre Jurimetria - Estatística aplicada ao Direito. No referido curso, aplicou-se parte da metodologia sugerida pela autora em sua pesquisa de doutorado. Foi solicitado aos alunos participantes que respondessem a um teste de sondagem para que se pudesse analisar o seu nível de Matemática Básica, necessária para o bom desempenho no curso. Tal teste foi elaborado usando-se os softwares $R$ e RStudio, também explorados ao longo das aulas. Em uma segunda etapa, sugeriu-se aos alunos que explorassem objetos de aprendizagem, o que notoriamente contribuiu para o andamento das aulas e entendimento dos conteúdos abordados.
\end{abstract}

Palavras Chave - Matemática Básica, RStudio, Shiny, Markdown, Jurimetria.

\section{Evaluation and automatic correction on the free software RStudio}

\begin{abstract}
This article provides foundation for creating documents for evaluation and automatic correction, exemplified on an experiment conducted in an extension course on jurimetrics - Statistics applied to the law. In that course, part of the methodology was applied, suggested by the author in her doctoral research. Participants were asked to students to answer a survey test so that it could analyze the level of Basic Mathematics, necessary for good performance in the course. This test was developed using the $R$ and RStudio softwares, also explored during the lessons. In a second step, it was suggested students to explore learning objects, which remarkably contributed to the progress of lessons and understanding of content covered.
\end{abstract}

Keywords - Basic Mathematics, RStudio, Shiny, Markdown, Jurimetrics.

\section{Introdução}

A Matemática, e mais especificamente a Estatística, podem ser grandes aliadas dos profissionais do Direito. A associação entre estas áreas tem suas raízes na tese de Nicolau Bernoulli I, publicada em 1709 tratando de temas diversos como precificação de seguros e confiança em testemunhas (BERNOULLI, 1709; HALD, 2003). O termo jurimetria foi apresentado em 1949 (LOEVINGER, 1949), buscando harmonia entre as ciências jurídicas e exatas com o intuito de trazer maior previsibilidade ao Direito utilizando a tecnologia vigente. Atualmente seus fundamentos são utilizados com frequência em seleções de jurados e comparações de cadeias DNA, sendo um caminho 
promissor para a elaboração legislativa e na construção de políticas públicas (ZABALA e SILVEIRA, 2014).

Sob esta perspectiva, a Pontifícia Universidade Católica do Rio Grande do Sul (PUCRS) ofereceu o primeiro curso de Jurimetria do Brasil, ministrado no primeiro semestre de 2016 na modalidade de extensão. O desafio era garantir que estudantes de Direito - em formação inicial e continuada, mas afastados da Matemática e suas aplicações - tivessem bom aproveitamento ao tratar dos conceitos exigidos ao longo do curso.

Com base em observações dos autores durante sua prática docente nas áreas de Matemática e Estatística, bem como em pesquisas já conhecidas nessas áreas (GIRALDO, 2004; HARDY, 2008; NASSER, 2009; LUZ, 2011), pode-se afirmar que os alunos que cursam disciplinas de cunho matemático nas Universidades apresentam muitas dificuldades relacionadas a conteúdos básicos, pré-requisitos para a disciplina em questão.

Com base na teoria da aprendizagem significativa de Ausubel (2003), considerou-se que os alunos matriculados no curso deveriam conhecer alguns prérequisitos (subsunçores) da área de Matemática, necessários para a aprendizagem significativa dos conceitos a serem trabalhados ao longo das aulas. Baseado nos testes propostos por Müller (2015), verificou-se o nível de Matemática Básica dos estudantes, adaptando-se as questões da autora a um instrumento que contemplasse os conteúdos necessários ao curso.

Implementou-se o instrumento no software livre RStudio (RSTUDIO TEAM, 2015), baseado nas bibliotecas shiny (CHANG et al, 2015) e markdown (ALLAIRE et al, 2015). A escolha desta tecnologia foi motivada para demonstrar a versatilidade do editor RStudio, que opera baseado na linguagem e ambiente R (R CORE TEAM, 2015) e escolhido para ser utilizado como apoio computacional ao longo das aulas de Jurimetria.

O nível dos alunos no teste inicial foi bastante satisfatório, com média 7.8 e desvio padrão 1.1. Direcionado pelas questões incorretas ou deixadas em branco, foram disponibilizados links para objetos de aprendizagem idealizados ou selecionados por Müller (2015), para que pudessem revisar os conteúdos envolvidos e acompanhar melhor a evolução do conteúdo do curso.

A partir da avaliação e depoimentos dos participantes, é possível afirmar que os objetos de aprendizagem mais uma vez contribuíram para o preenchimento de possíveis lacunas na aprendizagem dos subsunçores.

\section{Fundamentação Teórica}

A teoria da aprendizagem significativa foi utilizada ao longo desta proposta por se considerar que, para aprender significativamente tudo que envolve a Jurimetria, é necessário que se tenha uma boa base matemática, tendo-se alguns tópicos efetivamente como subsunçores nesse processo. 
Ao se fazer uma proposta que considere a importância de trabalhar matemática/estatística com estudantes ou profissionais do direito, tornou-se necessário identificar e preencher as lacunas na aprendizagem de conceitos de Matemática Básica.

Segundo Moreira (2011), o relacionamento da nova informação à estrutura cognitiva do aprendiz não é feito com qualquer aspecto dessa estrutura, mas com conhecimentos específicos, já existentes, que Ausubel chama de subsunçores.

Por outro lado, o que é incorporado à estrutura cognitiva é a substância do novo conhecimento, não apenas as palavras nas quais é expresso. Finalmente, "subsunçores" são conhecimentos já existentes na estrutura cognitiva do aprendiz, tais como proposições, concepções, ideias, invariantes operatórios ou representações sociais. Quando serve de âncora para um novo conhecimento, o subsunçor pode ser modificado, adquirindo novos significados (MOREIRA, 2011).

A aprendizagem significativa pode se apresentar em três diferentes tipos: aprendizagem representacional, conceitual e proposicional. Desataca-se aqui a aprendizagem conceitual, que está relacionada aos conceitos, ou seja, aos “objectos, acontecimentos, situações ou propriedades que possuem atributos específicos comuns e são designados pelo mesmo signo ou símbolo" (AUSUBEL, 2003, p. 2). A formação conceitual, segundo Ausubel, ocorre principalmente em crianças pré-escolares, enquanto que naquelas de idade escolar e nos adultos, ocorre a assimilação conceitual, visto que já há referentes, na estrutura cognitiva, para se combinarem e serem utilizados na definição de novos conceitos.

Um dos recursos sugeridos por Ausubel para resolver o problema da falta de subsunçores é o uso dos organizadores prévios. Organizadores prévios são materiais introdutórios, apresentados antes do material com o qual o professor pretende introduzir o novo conteúdo. Esses organizadores vão servir de "ponte" entre o que o aluno já sabe e o que ele deveria saber para poder aprender significativamente o novo conteúdo. Segundo Moreira (2011, p. 105), “[...] podem tanto fornecer 'ideias-âncora' relevantes para a aprendizagem significativa do novo material, quanto estabelecer relações entre ideias, proposições e conceitos já existentes na estrutura cognitiva e aqueles contidos no material de aprendizagem." Os organizadores prévios são recursos apresentados em níveis de abstração, generalização e inclusão mais altos do que o material a ser aprendido.

Na pesquisa realizada por Müller (2015) e adaptada no presente caso, utilizou-se como organizadores prévios objetos de aprendizagem que tratavam dos conteúdos elencados como subsunçores para a aprendizagem requerida e testados no questionário inicial.

De acordo com o IEEE (2000), um objeto de aprendizagem é definido como qualquer entidade, que pode ser utilizada, reutilizada ou referenciada durante o aprendizado apoiado por computador. Pode conter recursos variados, desde os mais simples, como um texto ou um vídeo, até alguns mais sofisticados, como um hipertexto, um curso ou até mesmo uma animação com áudio e recursos mais complexos.

Para Wiley (2000), objetos de aprendizagem são pequenos componentes instrucionais que podem ser reutilizados em diferentes contextos de aprendizagem. Cada objeto deve constituir-se de um módulo com conteúdo autoexplicativo, ou seja, que o aluno possa estudar sozinho, e estes módulos devem ser complementares, de 
forma que juntos formem um bloco mais completo de conteúdo. Além disso, o autor sugere que estes materiais devem ser digitais e reutilizáveis em diferentes situações e por diferentes grupos.

No âmbito educacional, tem-se observado que os objetos vêm sendo utilizados em larga escala, sendo um recurso muito importante para aulas a distância, mas também servindo como apoio ao ensino presencial. São recursos educacionais que podem ser utilizados para mediar e qualificar os processos de ensino e de aprendizagem. Devem ser materiais que motivem o estudante, através, por exemplo, da apresentação de situações-problema, que o desafiem e instiguem sua curiosidade.

Seguindo estas ideias, no caso dos alunos do curso de Jurimetria, procurou-se usar materiais e questões adaptados à sua realidade, explorando os conteúdos necessários de Matemática Básica.

\section{Implementação}

A implementação a seguir foi baseada em Attali (2015). Para instalar os ambientes R e RStudio, basta acessar https://www.R-project.org e http://www.rstudio.com, seguindo as orientações de acordo com cada sistema operacional. Após a instalação, recomendase adicionar alguns pacotes para a criação do documento interativo, inserindo os comandos em um arquivo chamado app.R no RStudio conforme Quadro 1.

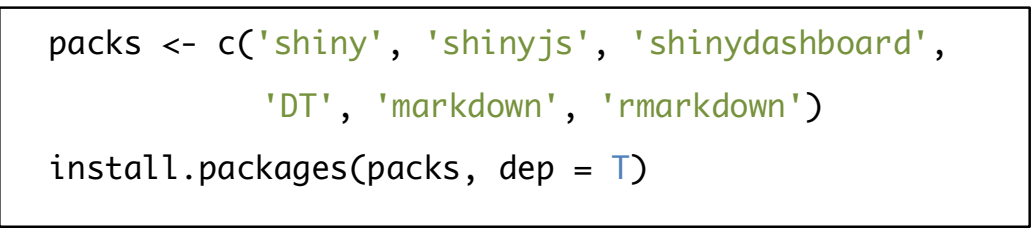

\section{Quadro 1 - instalação de pacotes para gerar documento interativo no RStudio}

Para gerar um arquivo HTML diretamente da programação em RStudio foi utilizada a biblioteca shiny (CHANG et al, 2015), desenvolvida com o princípio da reatividade, isto é, a capacidade de reagir às interações do usuário na tela do navegador. No Quadro 2 está apresentado o modelo mínimo de uma página shiny, que abre uma página HTML em branco no navegador. Apesar da aparente simplicidade, é um passo importante pela facilidade em associar diretamente um código $\mathrm{R}$ a um arquivo HTML.

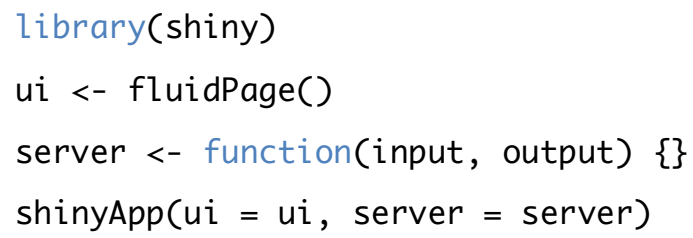

Quadro 2 - template básico de um aplicativo shiny 
Para melhorar a escrita simbólica foi utilizado o pacote markdown (ALLAIRE et al, 2015), uma sintaxe de formatação que converte a escrita de texto puro para HTML, incorporando ferramentas como a poderosa linguagem LaTeX (LAMPORT, 1994). Assim, as questões foram escritas separadamente em arquivos de extensão Rmd (R markdown), com sintaxe conforme Quadro 3 e saída simbólica conforme Figura 1.

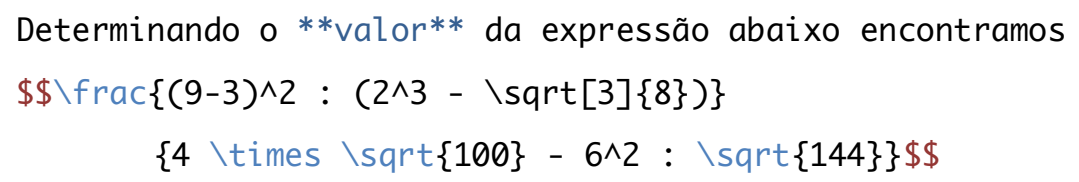

Quadro 3 - sintaxe markdown

$$
\begin{aligned}
& \text { Determinando o valor da expressão abaixo encontramos } \\
& \qquad \frac{(9-3)^{2}:\left(2^{3}-\sqrt[3]{8}\right)}{4 \times \sqrt{100}-6^{2}: \sqrt{144}}
\end{aligned}
$$

Figura 1 - saída simbólica em HTML

A chamada do arquivo Rmd pode ser realizada com as funções withMathJax e includeMarkdown aninhadas, conforme Quadro 4.

$$
\text { wi thMathJax(includeMarkdown('/caminho/questao.Rmd') }
$$

\section{Quadro 4 - funções aninhadas}

As alternativas foram apresentadas com a função radioButtons, que faz parte de um conjunto de funções para entrada de dados fornecidos pelo usuário. Pode-se apresentar os itens lado a lado (inline $=T$ ) ou um abaixo do outro (inline $=F$ ). A opção selected indica o item que ficará selecionado, conforme Quadro 5 e Figura 2.

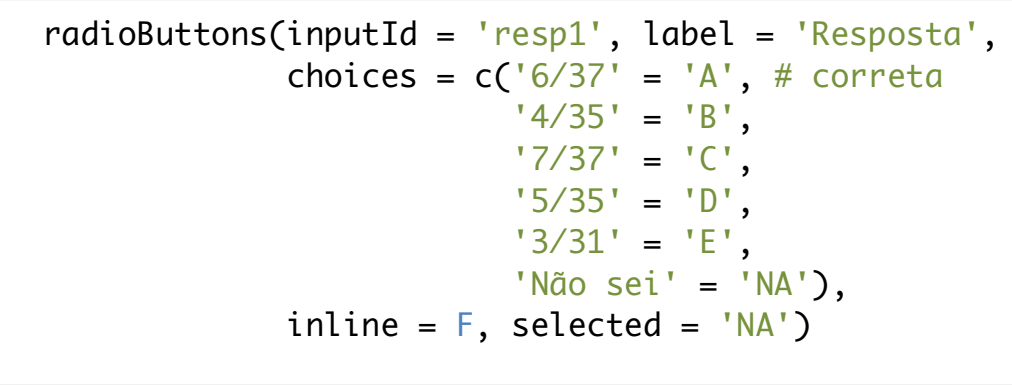

Quadro 5 - criação das alternativas com a função radioButtons

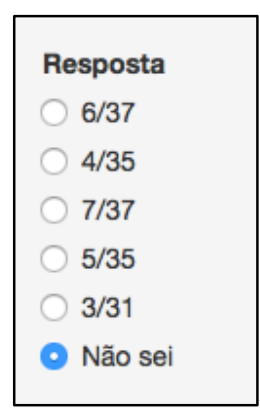

Figura 2 - interface das alternativas 
O armazenamento das variáveis foi realizado com a função saveData descrita no Quadro 6, que guarda o instante do armazenamento (hT) e as respostas em um arquivo csv (comma-separated values) nomeado genericamente como email_hT.csv.

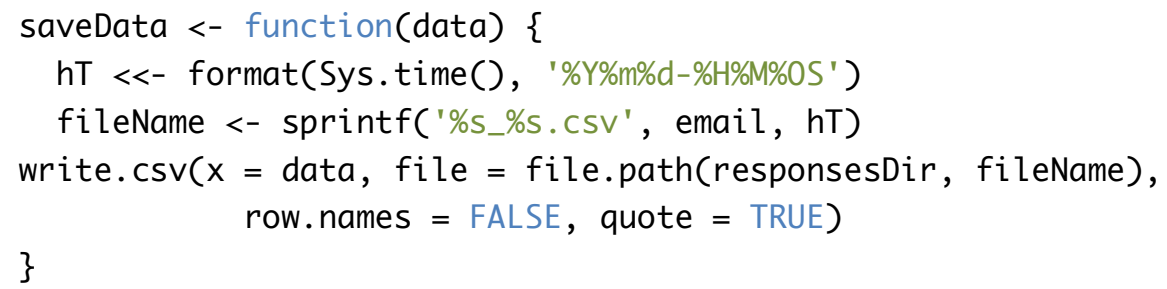

\section{Quadro 6 - função para salvar dados em arquivo}

Para a comparação das respostas do usuário com o gabarito, foi utilizada a função loadFi le, que chama o arquivo gerado por saveData e atribui a data.

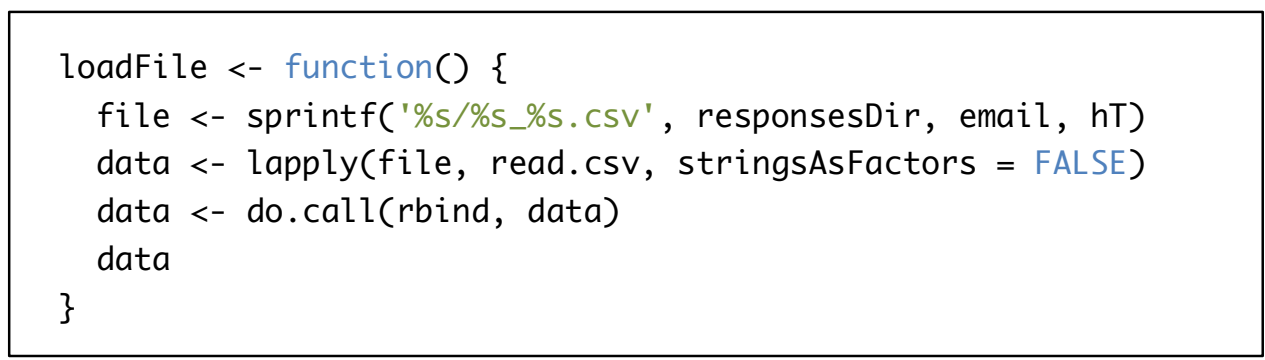

\section{Quadro 7 - função para carregar as respostas}

A pontuação é obtida comparando-se as respostas do usuário - armazenadas nas posições 3 a 12 de data - com o gabarito. São operadas dentro da classe de funções render e atribuídas a objetos dentro de output, como exemplificado no Quadro 8. 


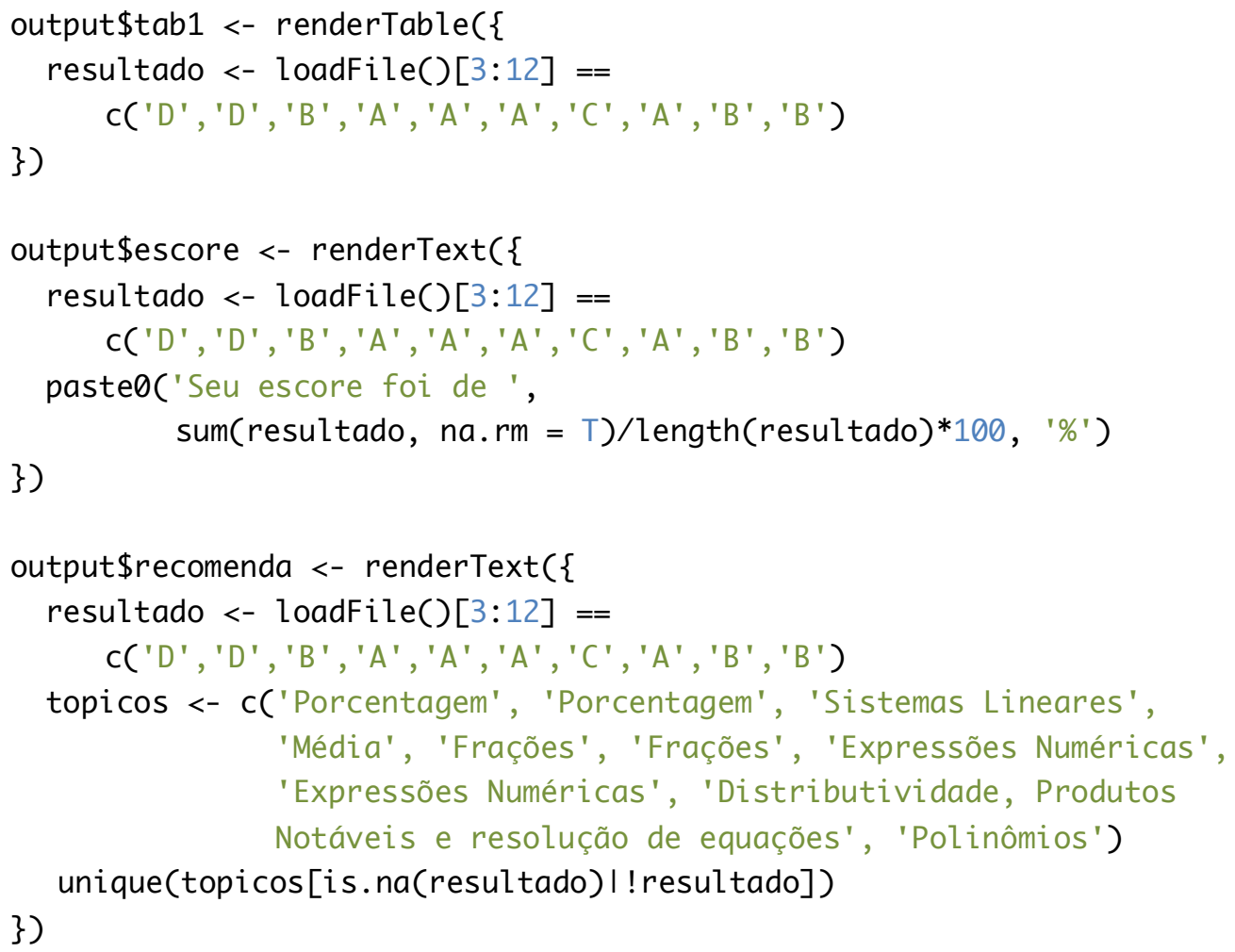

Quadro 8 - obtenção dos resultados

Pode-se adicionar um painel de título com a função titlePanel, que será mantido em todas as telas. Cada tela possui um botão de envio, ativado apenas quando o campo descrito em labelMandatory estiver preenchido.

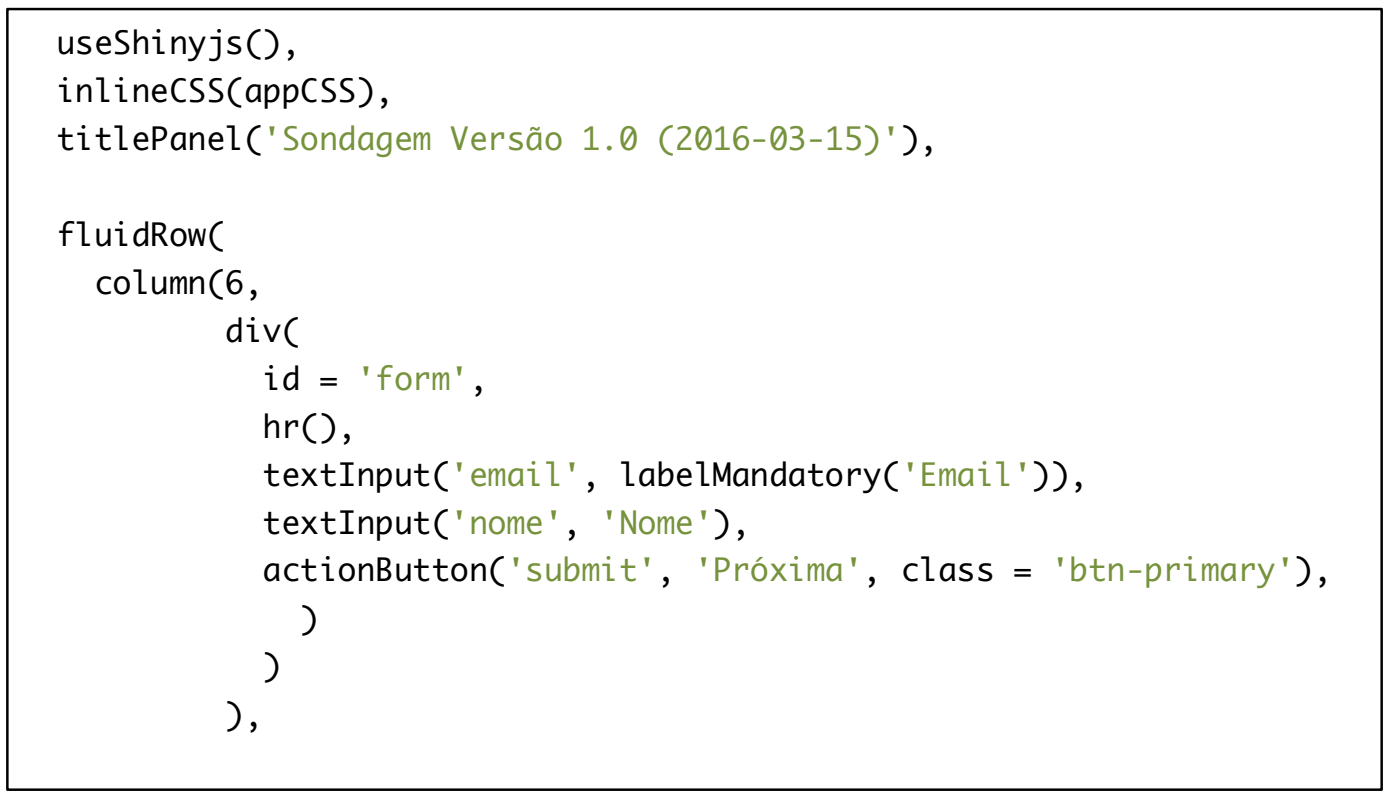


Como resultado obtém-se um arquivo HTML apresentado no navegador, com campos para serem preenchidos. $\mathrm{O}$ asterisco vermelho na Figura 3 indica um campo de preenchimento obrigatório. As questões são apresentadas em telas separadas, que avançam a cada clique no botão "Próxima".

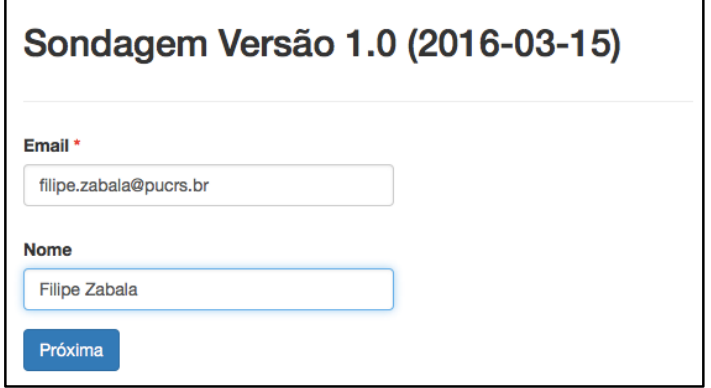

Figura 3 - aparência da primeira tela no navegador

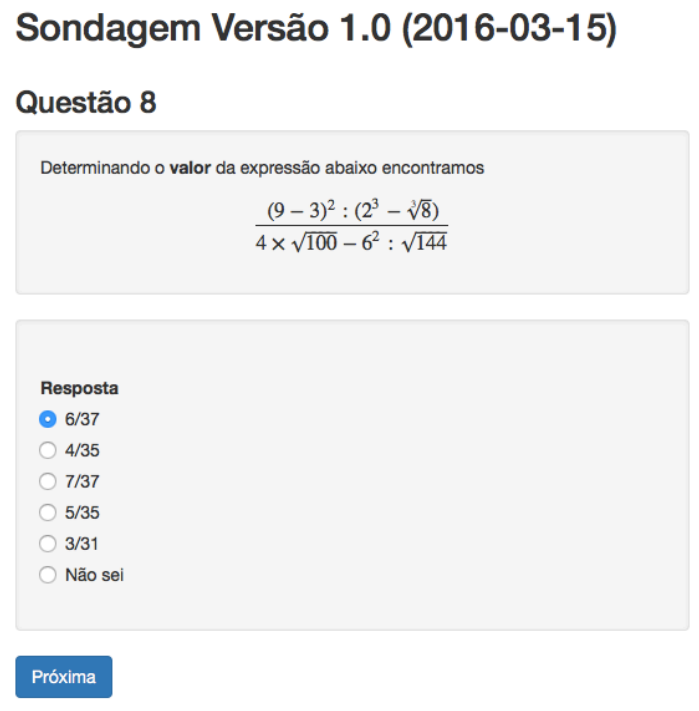

Figura 4 - aparência da questão 8 no navegador

$\mathrm{Na}$ tela final estão apresentados a tabela com as respostas, o escore e a sugestão de objetos de aprendizagem para consulta.

\section{Sondagem Versão 1.0 (2016-03-15)}

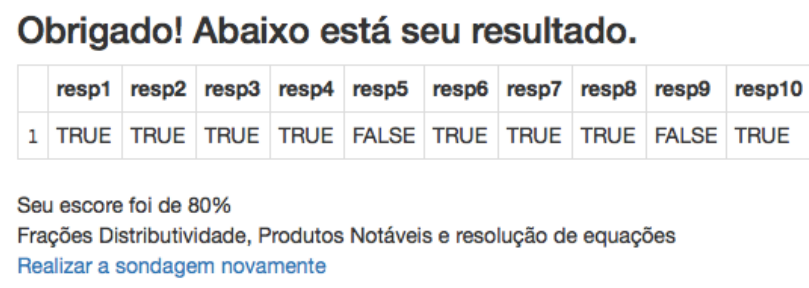

\section{Figura 5 - tela final do aplicativo}

Ao final é gerada uma tabela em formato csv no diretório desejado, contendo as informações do respondente.

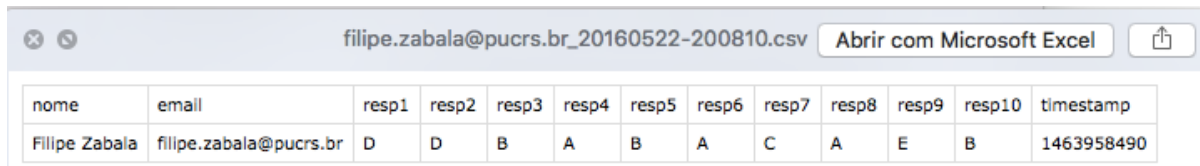

Figura 6 - tabela csv 


\section{Considerações Finais}

O presente artigo teve como objetivo compartilhar a implementação de uma ferramenta de avaliação e correção automática, baseada em software livre, e que pode ser adaptada a muitos contextos.

No caso aqui relatado, utilizando-se os procedimentos apresentados, criou-se um teste com conteúdos de Matemática Básica a fim de avaliar os conhecimentos dos participantes em um curso de Jurimetria. Tal teste foi aplicado no primeiro dia de aula, e enquanto os participantes do curso o respondiam, suas respostas eram armazenadas e validadas pelo próprio sistema.

De posse destas respostas, observou-se que os alunos já tinham um bom nível de Matemática Básica. Ainda assim, disponibilizou-se para o grupo links para objetos de aprendizagem, os quais os participantes afirmaram ter acessado com sucesso.

O instrumento construído mostrou-se bastante eficiente, uma vez que é baseado em uma ferramenta livre, de código aberto e capaz de armazenar e organizar os resultados obtidos. Além disso, proporciona feedback imediato ao estudante, uma vez seu desempenho é mostrado ao final da avaliação, bem como as questões acertadas ou erradas.

Sendo assim, acredita-se que esta ferramenta tem grande potencial no auxílio ao trabalho de professores e alunos, dados os resultados obtidos no curso aqui relatado e também os depoimentos dos alunos participantes, coletados na avaliação geral do curso.

\section{Referências Bibliográficas}

ALLAIRE J.J., HORNER J., MARTI V., PORTE N. markdown: 'Markdown' Rendering for $\mathbf{R}$. R package version 0.7.7. https://CRAN.R-project.org/package=markdown, 2015.

ATTALI, D. Mimicking a Google Form with a Shiny app. deanattali.com, 14 de junho de 2015. http://deanattali.com/2015/06/14/mimicking-google-form-shiny/

AUSUBEL, D. P. Aquisição e retenção de conhecimentos: uma perspectiva cognitiva. Lisboa: Plátano Edições Técnicas, 2003.

BERNOULLI, M. N. De usu artis conjectandi in jure. Basel, 1709.

CHANG W., CHENG J., ALLAIRE J.J., XIE Y, MCPHERSON, J. shiny: Web Application Framework for R. R package version 0.12.2. https://CRAN.Rproject.org/package=shiny, 2015.

GIRALDO, V. Descrições e conflitos computacionais: o caso da derivada. 2004. Tese (Doutorado em Engenharia de Sistemas e Computação) - Universidade Federal do Rio de Janeiro, Rio de Janeiro, 2004. 
HALD, A. A history of probability and statistics and their applications before 1750 , Vol. 501. John Wiley \& Sons, 2003.

HARDY, N. A subtle interplay between ordinary, algebraic and analytic registers in college level Calculus courses as a source of students' difficulties. In: INTERNATIONAL CONGRESS IN MATHEMATICAL EDUCATION (ICME 11), 11., 2008, Monterrey, Mexico. Proceedings...Disponível em: $<$ http://tsg.icme11.org/tsg/show/32>. Acesso em 20 de dezembro de 2012.

IEEE Learning Technology Standards Committee (LTSC), Draft Standard for Learning Object Metadata. Institute of Electrical and Electronics Engineers, 2000.

MÜller, T. J. Objetos de Aprendizagem Multimodais e Ensino de Cálculo: uma proposta baseada em análise de erros. 2015. Tese. (Doutorado em Informática na Educação) - Universidade Federal do Rio Grande do Sul, Porto Alegre, 2015.

LAMPORT, L. LaTeX user's guide and reference manual. Addison Welsley. Reading, Mass, 1994.

LOEVINGER, L. Jurimetrics - The Next Step Forward. Minnesota Law Review, v. 33, p. 455, 1949.

LUZ, V. M. da. Introdução ao Cálculo: uma proposta associando pesquisa e intervenção. 2011. Dissertação. (Mestrado em Ensino de Matemática) - Universidade Federal do Rio de Janeiro, Rio de Janeiro, 2011.

MOREIRA, M. A. Aprendizagem significativa: a teoria e textos complementares. São Paulo: Ed. Livraria da Física, 2011.

NASSER, L. Uma pesquisa sobre o desempenho de alunos de cálculo no traçado de gráficos. In: FROTA, M. C. R.; NASSER, L. Educação Matemática no ensino superior: pesquisas e debates. Recife: SBEM, 2009. p. 43-56.

R CORE TEAM. R: A Language and Environment for Statistical Computing. R Foundation for Statistical Computing, Vienna, Austria. https://www.R-project.org, 2015.

RSTUDIO TEAM. RStudio: Integrated Development Environment for R. RStudio Inc., Boston, MA. http://www.rstudio.com, 2015.

WILEY, D. A. Learning object design and sequencing theory. 2000. Tese (Doutorado em Filosofia) - Department of Instructional Psychology and Technology, Brigham Young University, Provo, UT, 2000.

ZABALA, F.J., SILVEIRA, F.F., Jurimetria: Estatística Aplicada ao Direito. Revista Direito e Liberdade, Natal, v. 16, n. 1, p. 73-86, jan./abr. 2014. 